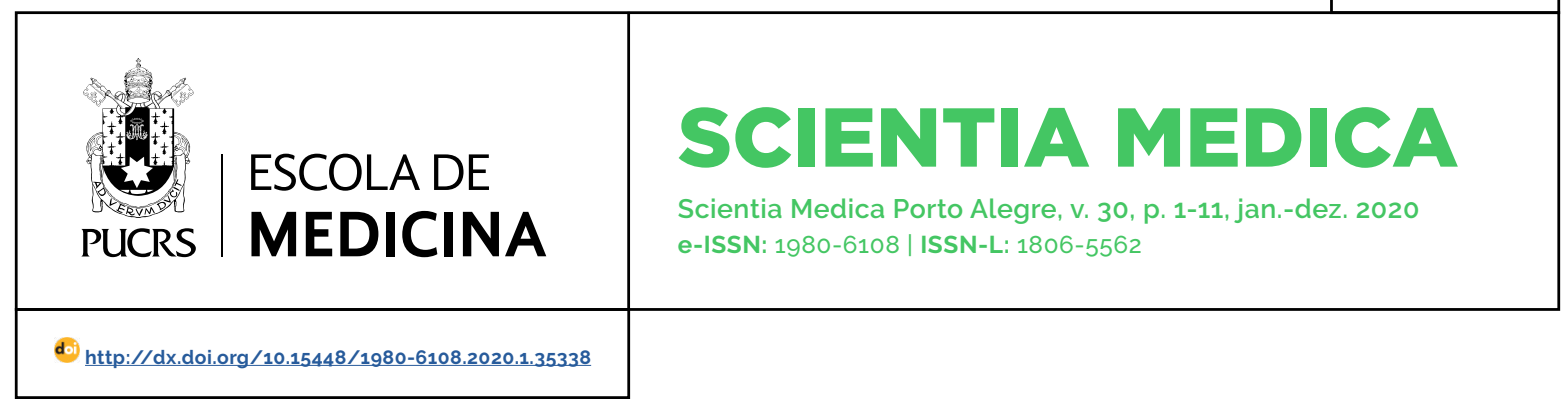

ARTIGO ORIGINAL

\title{
O impacto do uso da metformina para gestantes obesas na prevenção da pré-eclâmpsia
}

\author{
The impact of the use of metformine for obese pregnant women in prevention
}

\author{
Iramar Baptistella do \\ Nascimento ${ }^{1}$ \\ orcid.org/0000-0003-1268-2777 \\ iramar.nascimento@udesc.br
}

Willian Barbosa Sales ${ }^{2}$

orcid.org/0000-0003-2367-8271

willianbarbosasales@gmail.com

Guilherme Dienstmann² orcid.org/0000-0003-1268-2777 guidbio@gmail.com

\section{Matheus Leite Ramos de Souza ${ }^{2}$}

orcid.org/0000-0002-6297-6979 matheus.leite.souza@gmail.com

\section{Raquel Fleig ${ }^{3}$ \\ orcid.org/0000-0003-1934-6936 raquel.fleig@udesc.br \\ Jean Carl Silva² \\ orcid.org/0000-0002-3094-8180 \\ jeancarlsilva@gmail.com}

Recebido em: 16 ago. 2019.

Aprovado em: 16 nov. 2019.

Publicado em: 15 jul. 2020.

\section{(c) (i)}

Artigo está licenciado sob forma de uma licença Creative Commons Atribuição 4.0 Internacional.

\section{Resumo}

Objetivos: o artigo objetiva avaliar o uso de metformina em gestantes obesas na prevenção da pré-eclâmpsia.

Métodos: trata-se de um ensaio clínico randomizado com mulheres grávidas obesas com indice de massa corporal superior ou igual a $30 \mathrm{~kg} / \mathrm{m}^{2}$ divididos em dois grupos - um grupo de estudo, que utilizou metformina, e um grupo controle, que seguiu a rotina de pré-natal. As gestantes entraram no estudo com idade gestacional menor que 20 semanas e foram acompanhadas durante todo o periodo pré-natal. O diagnóstico de pré-eclâmpsia foi feito de acordo com os critérios da Organização Mundial de Saúde.

Resultados: verificou-se a redução de risco absoluto e o número necessário para tratar em um intervalo de confiança de 95\% para o resultado pré-eclâmpsia. Nos resultados, 272 gestantes foram avaliadas. No grupo de estudo $(n=127)$, oito $(6,3 \%)$ desenvolveram pré-eclâmpsia. No grupo controle $(n=145), 31(21,4 \%)$ tiveram a doença $(p<0,01)$.

Conclusão: o estudo apontou uma redução de risco absoluto de 15,1 vezes (IC95\%: 7,1-22,9) e número necessário para tratar de sete (IC95\%: 4,4-13,9). O uso de 1000 miligramas diárias de metformina indicou uma redução de 15,1 vezes no risco absoluto de pré-eclâmpsia na população estudada, com a necessidade de tratar sete gestantes para evitar um desfecho.

Palavras-chave: obesidade, gravidez, metformina, pré-eclâmpsia.

\section{Abstract}

Aims: The article aims to evaluate the use of metformin in obese pregnant women in the prevention of pre-eclampsia.

Methods: This is a randomized clinical trial with obese pregnant women with a body mass index greater than or equal to $30 \mathrm{~kg} / \mathrm{m}^{2}$ divided into two groups: a study group that used metformin and a control group that followed the prenatal routine. The pregnant women entered the study with gestational age less than 20 weeks and were followed throughout the prenatal period. The diagnosis of pre-eclampsia was made according to World Health Organization criteria.

Results: The absolute risk reduction and number needed to treat in a 95\% confidence interval for the preeclampsia result were checked. In the results, 272 pregnant women were evaluated. In the study group $(n=127)$ eight $(6.3 \%)$ developed preeclampsia, in the control group $(n=145) 31(21.4 \%)$ obtained the disease $(p<0.01)$ Conclusions: The study indicated an absolute risk reduction of 15.1 times (95\% Cl: 7.1-22.9) and number needed to treat of 7 (95\%Cl: 4.4-13.9). The use of 1000 milligrams daily metformin indicated a $15.1 \times$ reduction in the absolute risk of preeclampsia in the study population, with the need to treat seven pregnant women to avoid an outcome.

Keywords: obesity, pregnancy, metformin, preeclampsia. 
ABREVIATURAS: AAS, ácido acetilsalicilico; DMG, diabetes mellitus gestacional; IG, idade gestacional; IMC, Índice de massa corporal; IC, intervalo de confiança; MDV, Maternidade Darcy Vargas; NNT, número necessário a tratar; PIG, recém-nascido pequeno para a idade gestacional; $P E$, pré-eclâmpsia; RN, recém-nascido; RRA, redução de risco absoluto, RRA

\section{Introdução}

Na gestação as sindromes hipertensivas são merecedoras de grandes preocupações no cenário mundial, categorizadas como a primeira causa de morte no Brasil e a terceira no mundo (1). Estudos cientíicos demonstraram que o impacto do ganho de peso fora dos padrões adequados aumenta a incidência de pré-eclâmpsia (PE) durante a gravidez $(2,3)$.

Tanto a obesidade quanto a PE são enfermidades de origens multifatoriais $(4,5)$. A primeira envolve as dificuldades sobre o seu controle e sugere a necessidade de estratégias inovadoras mais eficientes para conduzir o ganho de peso de forma adequada durante a gestação (5,6). A outra, apresenta entre os diferentes critérios de diagnóstico para doença hipertensiva específica da gestação, os níveis de proteinúria elevados no periodo de 24 horas (7). Na literatura, estudiosos sugerem as disfunções cardiovasculares, a hipoxia na placenta e a dislipidemia materna como possiveis causas dessa afecção $(8,9)$. Portanto, devido à forte relação entre obesidade, dislipidemia, alterações vasculares e hipertensão tornou-se consistente a ideia sobre o comprometimento da obesidade com a PE $(10,11)$.

Pesquisas atuais com a administração de metformina têm demonstrado uma redução da PE através da sua ação sobre os receptores correspondente ao fator de crescimento endotelial vascular $(9,12)$. Outros tratamentos com ácido acetilsalicilico (AAS), dipiridamol, heparina ou ozagrel são discutidos na literatura e utilizados na gravidez para efeitos profiláticos fisiológicos envolvendo anormalidades na coagulação e possiveis alterações que competem a associação entre prostaciclina A2 e tromboxane. No entanto, os prognósticos profiláticos refletiram em uma atenuada prevenção às incidências de doença hipertensiva especifica da gestação em diferentes estudos (13,14).

Portanto, uma vez que estudiosos sugerem relações da metformina com a redução do ganho de peso e um melhor equilibrio metabólico no sistema cardiovascular hipotetizamos a ideia de que o cloridrato de metformina poderá ser promissor na prevenção da PE em grávidas com o índice de massa corporal $(I M C) \geq 30(9,15)$. Desta forma, o estudo tem por objetivo avaliar o uso da metformina para gestantes obesas na prevenção da PE.

\section{Materiais e métodos}

Foi realizado um estudo de ensaio clínico randomizado com gestantes obesas sem diagnóstico de qualquer tipo de diabetes. $O$ estudo foi desenvolvido no periodo de 31 de outubro de 2014 a 31 de dezembro de 2017. Esta pesquisa é parte de uma pesquisa maior com a utilização da metformina para prevenção do Diabetes Mellitus Gestacional (DMG). O tratamento foi desenvolvido na Maternidade Darcy Vargas (MDV), localizada no municipio de Joinville, estado de Santa Catarina, Brasil. A MDV possui serviço multidisciplinar de atendimento às gestantes obesas caracterizadas como grupo de risco. As gestantes são encaminhadas pela Unidade Básica de Saúde para o Ambulatório da MDV.

Para o cálculo da amostra, considerou-se uma incidência de $14 \%$ de PE em gestantes obesas com o IMC $(\geq 30,0)$. O presente estudo teve por propósito uma redução de $42,9 \%$, ou seja, de $14 \%$ para $6 \%$ com poder de $80 \%$ e erro alfa de 0,05. Desta forma, de acordo com o cálculo, seria necessário uma amostra de 121 gestantes obesas para cada grupo.

A pesquisa foi aplicada conforme regulamentações da Resolução 466/12 do Conselho Nacional de Saúde. O sigilo das gestantes e de seus filhos foi preservado. O estudo foi aprovado de acordo com a Plataforma Brasil, pelo número CAAE 34863514.1.0000.5366. As gestantes foram devidamente orientadas e esclarecidas quanto aos objetivos e informadas sobre o direito de recusa à participação em qualquer momento da pesquisa em questão, sem que, para tanto, sofressem prejuizo ou penalidade de qualquer natureza, quer seja para ela ou para o seu bebê. Acrescente-se que foi mantida a integridade, garantia e privacidade das participantes. 
Concomitantemente a confirmação do número correspondente ao Registro Brasileiro de Ensaios Clínicos, número: U1111-1162-6908. Este relatório segue as recomendações das Normas Consolidadas do Relatório de Avaliação (CONSORT) (16).

As grávidas com idade gestacional (IG) $\leq 20$ semanas e IMC $\geq 30 \mathrm{~kg} / \mathrm{m}^{2}$ foram encaminhadas pela Unidade Básica de Saúde para o Ambulatório de Obesidade Gestacional da MDV. Os pacientes foram convidados a participar de palestras realizadas no auditório do MDV sobre a obesidade gestacional e a eficácia da metformina na prevenção de riscos materno-fetais, durante a qual os objetivos do estudo, bem como seus riscos e benefícios. As gestantes que concordaram em fazer parte da pesquisa assinaram duas vias de consentimento livre e esclarecido. As palestras ocorreram às quintas-feiras, às 7 h 30 minutos, durante todo o estudo.

Foram incluidas todas as gestantes com diagnóstico de obesidade, conforme critérios da Organização Mundial de Saúde, com IMC $\geq$ $30 \mathrm{Kg} / \mathrm{m}^{2}$, somente as grávidas de etnia branca, com idade igual ou superior a 18 anos, gestação única, cesárias primárias ou grávidas obesas que já haviam realizado o procedimento em gestações anteriores, IG inferior a 20 semanas de gestação com administração da metformina desde o ingresso no estudo até o parto. As gestantes obesas, para serem incluídas deveriam se apresentar com as seguintes características: não fumantes, sem história familiar de hipertensão e pré-eclâmpsia, sem qualquer tipo de diabetes ou presença de doença hepática, renal, gastrointestinal, alergia importante a medicamentos, ou outra característica que prejudicasse a absorção, distribuição, excreção ou o metabolismo do fármaco. A gestante não poderia estar administrando nenhum outro medicamento que pudesse interferir nos desfechos hipertensivos, dentre outros, o AAS e dosagens elevadas de ácido fólico. A exclusão foi realizada através de fatores correspondentes à perda de seguimento, ou seja, por não aderirem ao tratamento; abandono da pesquisa ou por intolerância à droga.

O acompanhamento foi realizado durante o pré-natal, conforme rotina básica recomendada pelo Ministério da Saúde. Todas as gestantes da pesquisa receberam assistência padrão da maternidade, sendo atendidas pelos serviços de nutrição, enfermagem, fisioterapia e obstetrícia. A MDV utiliza orientação de dieta com pequenas reduções na ingestão calórica de $24 \mathrm{kcal} / \mathrm{kg} /$ dia, com dieta fracionada em cinco a seis refeições diárias, com composição calórica diária incluindo 40-50\% de carboidratos complexos ricos em fibras, $20 \%$ de proteinas e $30-40 \%$ de gorduras não saturadas. A distribuição calórica diária foi de $10-20 \%$ no café da manhã, 20-30\% no almoço, 20-30\% no jantar e até 30\% para lanches, incluindo um lanche ao deitar-se, para evitar a hipoglicemia noturna. Quanto às atividades físicas, as grávidas também receberam orientações dos profissionais especializados da maternidade, com programa de caminhadas regulares de 20 minutos por dia (17).

No grupo metformina as gestantes receberam, junto com o tratamento padrão, a metformina, com dosagem diária de 1000 miligramas (mg/d): 500 mg no café e 500 mg no jantar. No grupo controle, somente o tratamento padronizado do hospital. A dosagem de $1000 \mathrm{mg} / \mathrm{d}$ foi por consenso da equipe de pesquisa com o objetivo de verificar uma dosagem inferior a já utilizada em outros estudos científicos que utilizaram a metformina em gestantes obesas não diabéticas $(12,18)$. A intenção foi observar os resultados da PE em menor dosagem possibilitando o mínimo de intolerância ao medicamento e aumentar ainda mais a aceitabilidade do medicamento por parte da mulher grávida, como observado em um estudo precedente (15). No entanto, a sensibilidade à droga não foi o principal objetivo desta pesquisa.

Durante a primeira visita ao ambulatório de obesidade gestacional, os participantes foram randomizados com um algoritmo computadorizado utilizando o programa Microsoft Excel (Microsoft, Redmond, WA, EUA), que gerou uma lista de pedidos de alocação aleatória em uma proporção não fixa, dividida em dois grupos: um grupo de estudo, que foi tratado com metformina e recebeu orientação sobre dieta e atividade física, e um grupo controle, que recebeu apenas aconselhamento sobre dieta e atividade física.

As participantes receberam um selo codificado 
em seu registro de acompanhamento pré-natal, que as identificou como participantes da equipe de pesquisa multidisciplinar durante as visitas ambulatoriais ao longo do estudo. Todas as pacientes foram identificadas utilizando um formulário de pesquisa específico contendo o nome da participante; data de nascimento; idade da gestante; estado civil; ocupação; nível educacional; etnia; IMC; IG; alergia à metformina; intervalo entre entregas; aborto; tipo de entrega; número de gestações; idade ao nascimento do primeiro filho; uso de medicação durante a gravidez; diagnóstico de doença renal, hepática ou gastrointestinal; e diagnóstico de DMG.

Sobre a coleta de dados, ocorreu da seguinte forma: idade da mãe, estado civil, escolaridade, número de gestações, IG de entrada e IMC nos três trimestres de gestação (chegada, no segundo trimestre entre 24 a 28 semanase, no terceiro trimestre gestacional, com 33 a 35 semanas). Também se identificou o DMG em números absolutos e relativos na comparativa entre os dois grupos.

Vale ressaltar que o principal objetivo deste estudo foi analisar o percentual e a redução de PE em gestantes obesas que fizeram uso da metformina durante a gestação através dos parâmetros estatísticos sobre redução de risco absoluto (RRA) e número necessário a tratar (NNT). Nos desfechos secundários, as buscas das informações foram nos prontuários dos recémnascidos das respectivas gestantes. Optou-se por verificar a razão de chances (odds ratio) para prematuridade, quando a mãe gerou seu recémnascido <37 semanas, recém-nascidos pequenos para a idade gestacional (PIG), Apgar no $1 .^{\circ}$ e $5^{\circ}$ minuto e unidade de terapia intensiva neonatal. O peso do recém-nascido foi categorizado pela curva de Battaglia e Lubchenco, por ser uma das curvas mais utilizadas nas maternidades latino-americanas (19). Nessa curva, as crianças são classificadas como PIG, aquelas abaixo do percentil 10, adequadas para a idade gestacional, que estão entre os percentis 10 e 90 e grandes para a idade gestacional, que estão acima do 90. ${ }^{\circ}$ percentil. Por outro lado, o ganho de peso materno não foi coletado, uma vez que não era rotina do serviço, apenas no IMC trimestral.
As variáveis foram tratadas estatisticamente por meio do software IBM SPSS Statistics 21. A principio, todos os dados foram analisados descritivamente. Para as variáveis contínuas, a análise foi realizada por meio do cálculo de médias e desvios-padrão. Para as análises de hipótese e de comparação, entre as médias dos grupos foi utilizado o teste $\mathrm{t}$ de Student e, quando a normalidade foi rejeitada utilizouse o teste não paramétrico de Mann-Whitney. Como suporte para verificar a normalidade da população foi utilizado o teste KolmogorovSmirnov. Para se testar a homogeneidade dos grupos em relação às proporções foi aplicado o teste qui-quadrado ou o teste exato de Fisher para frequências menores que cinco. Os fatores de risco foram identificados por análise univariada, que comparou as variáveis categorizadas pelo teste do qui-quadrado. Calculou-se a RRA e NNT para PE. O RRA é o resultado após a intervenção, ou seja, a prevalência do risco basal da amostra que se mantém constante e vai reduzir após a intervenção desenvolvida. O NNT é o número de pacientes que se tem a tratar com determinado fármaco para se evitar um desfecho da doença (20). Para os desfechos dos RN, instituiu-se o modelo de regressão logística multinomial contendo valores ajustados para o cálculo de razão de chances (odds ratio). Optou-se por regressão logística multinomial devido a um maior número de variáveis categóricas. Para todos os resultados da pesquisa adotou-se um intervalo de confiança (IC) de 95\%, considerandose valores significativos quando $\mathrm{p}<0,05$. As variáveis ajustadas foram: idade da gestante, estado civil, escolaridade, número de gestações, IG de entrada, IMC gestacional, desenvolvimento de PE, DMG, nascimento de RN prematuro, nascimento de RN PIG, ocorrência de Apgar ao $1 .^{\circ}$ e $5 .^{\circ}$ minutos baixos (<7) e internação em UTI neonatal. O escore Apgar corresponde à avaliação de cinco itens do exame físico do RN, com 1, 5 e 10 minutos de vida e, quando o escore é $<7$ pode ser indicativo de alterações no seu estado geral e de vitalidade. Foram considerados baixos escores de Apgar valores menores do que sete. 


\section{Resultados}

A Unidade Básica de Saúde enviou 340 gestantes obesas com IMC $\geq 30\left(\mathrm{~kg} / \mathrm{m}^{2}\right)$, caracterizadas como gestação de alto risco, para o Ambulatório de Obesidade para atendimento especializado da MDV. No presente estudo, 32 gestantes apresentaram caracteristicas incompativeis com os requisitos pré-estabelecidos: nove gestantes fizeram cirurgia bariátrica, sete eram tabagistas, seis estavam administrando outros medicamentos que poderiam influenciar nos desfechos, quatro tiveram experiência prévia com doenças gastrointestinais (dispepsia, gastroenterite, esofagite ou gastrite), cinco com doenças renais (história de pielonefrite, cistos renais ou insuficiência renal) e uma gestante já estava ciente de sua hipersensibilidade aos medicamentos. Desta forma, 308 pacientes apresentaram critérios adequados para participar do projeto, 10 se recusaram a participar, restando 298 gestantes para a randomização. Dessas, 149 gestantes no grupo controle e 149 no grupo intervenção com metformina. Durante o tratamento, quatro gestantes do grupo controle abandonaram a pesquisa por motivos não justificados e partiram antes de 22 semanas. No grupo metformina, cinco abandonaram a pesquisa por apresentarem náuseas, vômitos e diarreia frequentes após a administração do medicamento e 17 por motivos não justificados, e partiram antes de 22 semanas, ou seja, não deram à luz a seus bebês na MDV. Dessa forma, 272 gestantes foram incluídas para análise, realizaram todo o pré-natal e ganharam seus bebês na MDV. Dessas, 145 $(53.3 \%)$ gestantes do grupo controle e $127(46,7 \%)$ do grupo metformina, mais detalhado na Figura 1.

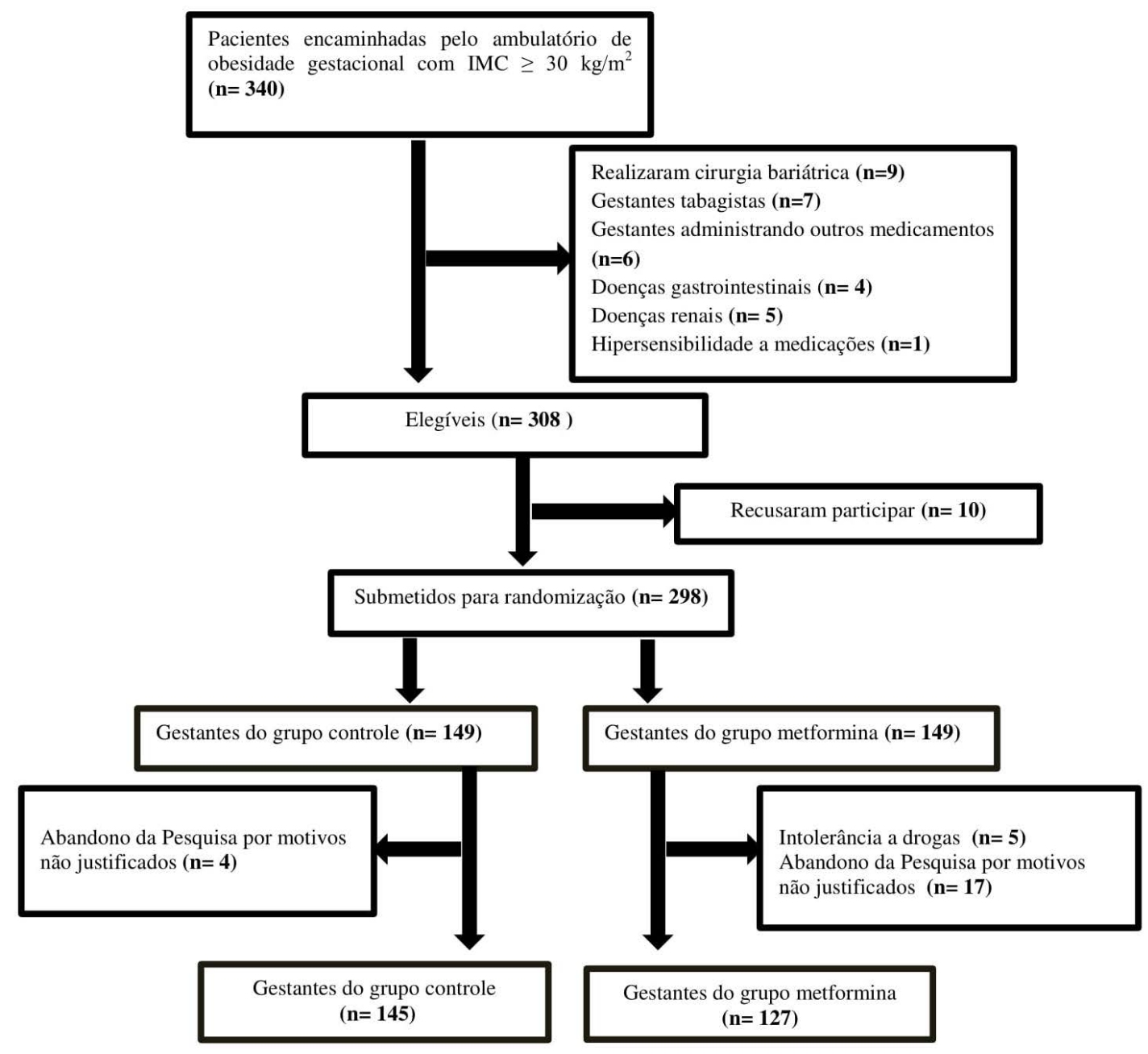

Figura 1 - Fluxograma dos participantes em cada estágio do estudo 
$6 / 11$ Scientia Medica Porto Alegre, v. 30, p. 1-11, jan.-dez. 2020 | e-35338

A Tabela 1 apresenta as características gerais das gestantes em uma comparativa entre os dois grupos: controle e metformina. As variáveis qualitativas não apontaram diferenças para os fatores relacionados ao estado civil, escolaridade e número de gestações anteriores $(p>0,05)$. Nos aspectos quantitativos não houve diferença quanto a idade das mães, o número de gestações, o IMC gestacional e o DMG nos resultados estatísticos ( $p>0,05)$. Porém, a IG de entrada no projeto revelou uma diferença estatística que talvez possa vir a influenciar no tratamento $(p<0,01)$.

TABELA 1 - Caracteristicas gerais das pacientes

\begin{tabular}{|c|c|c|c|c|}
\hline & Amostra total & Grupo Controle & Grupo Metformina & $\mathbf{P}$ \\
\hline $\mathrm{N}$ & 272 & 145 & 127 & \\
\hline Idade materna, média $\pm D P$ & $29,3 \pm 6,0$ & $29,6 \pm 6,1$ & $29,1 \pm 5,9$ & $0,50^{*}$ \\
\hline \multicolumn{5}{|l|}{ Estado civil, n (\%) } \\
\hline Solteira & $42(15,4)$ & $24(16,6)$ & $18(14,2)$ & \multirow{3}{*}{$0,56^{+}$} \\
\hline Casada & $205(75,4)$ & $110(75.9)$ & $95(74,8)$ & \\
\hline Outro & $25(9,2)$ & $11(7,6)$ & $14(11,0)$ & \\
\hline \multicolumn{5}{|l|}{ Escolaridade, n (\%) } \\
\hline$<8$ anos & $41(15,1)$ & $24(16,6)$ & $17(13,4)$ & \multirow{4}{*}{$0,42^{+}$} \\
\hline Fundamental completo & $80(29,4)$ & $37(25,5)$ & $43(33,9)$ & \\
\hline Médio complete & $123(45,2)$ & $70(48,3)$ & $53(41,7)$ & \\
\hline Superior complete & $28(10,3)$ & $14(9,7)$ & $14(11,0)$ & \\
\hline$N^{0}$ de gestações, média $\pm \mathrm{DP}$ & $2,8 \pm 2,2$ & $2,9 \pm 2,2$ & $2,6 \pm 2,1$ & $0,24^{*}$ \\
\hline \multicolumn{5}{|l|}{ Histórico gestacional, n (\%) } \\
\hline Primigestas & $28(10,3)$ & $12(4,4)$ & $16(5,9)$ & $0,16^{+}$ \\
\hline Multigestas & $244(89,7)$ & $133(48,9)$ & $111(40,8)$ & $0,24^{+}$ \\
\hline IG de entrada, média $\pm D P$ & $12,4 \pm 4,9$ & $11,6 \pm 4,7$ & $13,1 \pm 5,1$ & $0,01^{*}$ \\
\hline IMC chegada, média $\pm D P$ & $37,6 \pm 4.8$ & $37.5 \pm 4.7$ & $37,7 \pm 4,9$ & $0.26^{\ddagger}$ \\
\hline $\mathrm{IMC} 3^{\circ}$ trimestre, média $\pm \mathrm{DP}$ & $38,5 \pm 5,0$ & $38,4 \pm 4,9$ & $38,6 \pm 5,1$ & $0.49^{\ddagger}$ \\
\hline DMG, n (\%) & $21(7,2)$ & $13(9,7)$ & $8(4,6)$ & $0,14^{+}$ \\
\hline
\end{tabular}

DMG, diabete melito gestacional; DP, desvio padrão; IG, idade gestacional; IMC, indice de massa corporal. " teste t de student; ${ }^{+}$qui-quadrado; ${ }^{\ddagger}$ Mann Whitney.

A Tabela 2 representa os itens avaliados nos desfechos neonatais: via de parto, prematuridade, peso do RN, PIG ou grandes para a idade gestacional, Apgar $1 .^{\circ}$ e $5 .^{\circ}$ minuto e o número de óbitos. Os parâmetros estatísticos não revelaram diferenças entre os grupos avaliados. 
TABELA 2 - Desfechos neonatais observados no grupo controle e no grupo de metformina

\begin{tabular}{ccccc}
\hline Desfechos & $\begin{array}{c}\text { Grupo Controle } \\
\mathbf{n}(\%)\end{array}$ & $\begin{array}{c}\text { Grupo Metformina } \\
\mathbf{n}(\%)\end{array}$ & $\begin{array}{c}\text { OR ajustado } \\
\text { (IC95\%) }\end{array}$ & P \\
\hline Prematuridade & $9(4,8)$ & $9(5,3)$ & $1,68(0,55-5,14)$ & 0,35 \\
Apgar 1' $<7$ & $13(7,0)$ & $14(8,2)$ & $1,43(0,63-3,24)$ & 0,39 \\
Apgar 5' $<7$ & $1(0,5)$ & $0(0,0)$ & - & $\ldots$ \\
UTI neonatal & $3(1,6)$ & $3(1,8)$ & $1,89(0,27-13,17)$ & 0,52 \\
\hline
\end{tabular}

IC95\%, intervalo de confiança de 95\%; OR, odds ratio; PIG, pequeno para idade gestacional; UTI, unidade de terapia intensiva.

Sobre a razão de riscos referentes à utilização da metformina para prevenção da PE, o grupo que administrou a metformina ( $n=127)$ apresentou a incidência de oito $(6,3 \%)$ de PE e, no grupo controle ( $n=145), 31$ (21,4\%) tiveram desfecho de PE, os parâmetros mostraram valores significativos ( $p<0,01$ ). A RRA foi de 15,1 vezes (IC95\%: 7,1- 22,9) com NNT de 7,0 (IC95\%: 4,4 - 13,9).

\section{Discussão}

Este estudo mostrou o impacto da utilização do cloridrato de metformina em gestantes obesas para prevenção da PE. Foi possivel avaliar o uso da metformina na incidência da doença, quantificar a RRA, o NNT. Uma pesquisa publicada em 2004 mostrou uma forte relação do IMC aumentado com hipertensão crônica e PE durante a gravidez (21). Contudo, independentemente do prognóstico benéfico a se adquirir com a administração de uma droga, torna-se essencial o incentivo à prática de dieta e exercicio físico (17).

Dentre os resultados encontrados, a média de idade das mães, o estado civil, a escolaridade e o número de gestações anteriores não apontaram diferenças entre os dois grupos avaliados. Todavia, são fatores que em outras pesquisas atestaram percentuais relevantes a incidências de PE, demarcando as mães mais jovens, e a baixa escolaridade e, até mesmo, as relações conjugais, que se observou serem variáveis que influenciam no número de assistências pré-natais, paridade, obesidade e hábitos de vida $(22,23)$.

Devido ao fato de que as estimativas na literatura cientifica mostram 82,8\% da incidência de
PE em mães primigestas com etnias não brancas (24), o presente estudo, para evitar enganos, visou experimentar o uso da metformina apenas gestantes da raça branca. Vale ressaltar que devido aos reflexos da colonização europeia, que se estabelece com maior representação em nossa população na região Sul do Brasil, prevalece uma grande quantidade de gestantes da raça branca.

O presente estudo não apontou valores reduzidos para o DMG e para o IMC gestacional com o uso da metformina. Todavia, a metformina implica em uma opção coadjuvante, uma vez que estudiosos identificaram que o antidiabético pode ser uma possivel contribuição na redução do IMC, que por sua vez, apresentou relações com a hiperlipidemia e doença hipertensiva especifica da gestação (24). A literatura sugere que a metformina reduz a produção de fatores angiogênicos SFlt1e a endoglina através da sua ação sobre os receptores concernentes ao fator de crescimento endotelial vascular solúvel-1 e endoglina solúvel, favorecendo a melhorias nas disfunções angiogênicas $(9,25)$. Já a profilaxia da PE com AAS tem por função inibir a ação da enzima cicloxigenase reduzindo a produção de tromboxano, um lipídio que favorece a agregação plaquetária da mãe grávida (26).

Pesquisadores evidenciaram redução da doença em mães que tomaram metformina a partir de 12 a 18 semanas de gravidez (12). A nossa média de início quanto ao uso da droga se estabeleceu entre 11 a 15 semanas, resultados esses que preconizam dar começo à administração do medicamento em periodos anteriores a 20 semanas de gestação. 
A dosagem a ser administrada tornou-se relevante na concepção sobre a profilaxia da PE. A presente pesquisa utilizou $1000 \mathrm{mg} / \mathrm{d}$ na prevenção da doença. Estudo similar com gestantes obesas utilizou $1000 \mathrm{mg} / \mathrm{d}$ nos primeiros sete dias com aumento de $0.5 \mathrm{~g}$ por semana até uma dose máxima de $3000 \mathrm{mg} / \mathrm{d}$ na quinta semana até o parto [12].

Dentre os efeitos colaterais mais frequentes com o uso da metformina encontrou-se a intolerância ao medicamento (27). No presente estudo, dentre as 149 gestantes que estavam administrando a metformina com a dosagem de 1000 mg/d, apenas cinco $(3,4 \%)$ tiveram intolerância à droga com manifestações de náuseas, vômitos, diarreia.

Sobre as intervenções com outras drogas, recomenda-se que a aspirina comece entre 12 e 14 semanas de gestação e, nas descobertas científicas de maior impacto, ela se limitou aos benefícios preventivos na incidência de PE em mulheres com risco moderado ou grave (28). Da mesma forma, ensaios de campo usando dipiridamol, heparina ou ozagrel não revelaram valores expressivos (14). Vale ressaltar que, embora a aspirina seja frequentemente usada na gravidez, ela é risco C na Classificação da Food and Drug Administration. Resultados recentes demonstraram que é segura tanto para a mãe como para o feto, quando administrada em baixas doses (< $150 \mathrm{mg} / \mathrm{dia}$ ), durante toda a gestação (29,30). O uso crônico ou intermitente em altas doses (> $325 \mathrm{mg} / \mathrm{dia}$ ) deve ser evitado, principalmente, próximo ao termo, pois pode causar fechamento prematuro do ducto arterioso, comprometimento da coagulação, tanto materna como no neonato, além de intoxicação congênita por salicilato (31). É excretada pelo leite em baixas doses e efeitos adversos na função plaquetária do lactente não têm sido descritos. A recomendação é de que seu uso na amamentação seja realizado com cautela (31). Em estudos com animais, seus efeitos são controversos, com chances de alterações morfológicas no feto, na placenta e na diminuição do ganho de peso materno (32).

Um estudo de revisão com 59 ensaios utilizando AAS demarcou uma redução no risco da PE de
17\%, (RR=0,83; IC95\%=0,77-0,89), com NNT de 72 mulheres grávidas com a administração de aspirina. Destacaram-se as mulheres de médio e alto risco à $\mathrm{PE}$, os valores redutivos para o RRA foram mais convincentes, apresentando o NNT de 19 para as gestantes de alto risco, comparado com o NNT de 119 para as de baixo risco (33). O estudo aqui apresentado demarcou uma RRA de 15,1 vezes (IC95\%:7,1-22,9) para incidência de PE em mães grávidas obesas, uma vez que é necessário tratar sete gestantes a fim de prevenir um desfecho da enfermidade (NNT=7).

Desta forma, a metformina parece ter alcançado melhores favorecimentos quanto aos seus preditores, uma vez que a utilização da aspirina esteve em contrapartida de resultados em diferentes pesquisas de ensaio clínico e, concomitantemente, independentemente do periodo que se estabeleceram as intervenções com AAS, antes ou após 16 semanas de gestação, os seus efeitos apresentaram um impacto limitado ou nenhuma eficácia (34). Outros estudos não indicaram valores estatisticamente significativos para a redução da doença e, ao mesmo tempo, aumentaram as chances de envolvimento materno (35). Portanto, a metformina sugere maior atenção em seu uso e aceitação por parte da população gestacional.

Na estimativa sobre o desfecho do RN, as análises não resultaram em dados significantes para nenhuma das variáveis em questão. Todavia, mesmo que a metformina não tenha demonstrado uma redução nos desfechos aqui avaliados, a droga confirmou segurança após a sua administração. Semelhante aos resultados de pesquisas anteriores, que além de ratificarem uma melhor aceitação da metformina pelas gestantes, os pesquisadores não atestaram riscos nos respectivos bebês (15).

Sobre as limitações, houve um pequeno número de estudos científicos utilizando a metformina para a prevenção de PE em gestantes obesas sem o diagnóstico de diabetes. O ponto forte foi o apoio dos técnicos do setor administrativo e da equipe de enfermagem da maternidade para com o acesso aos protocolos da maternidade facilitando a coleta de dados. Outro fator positivo foram as 
diferentes pesquisas cientificas que focalizam o equilibrio angiogênicos e antiangiogênico de âmbito placentário em que a atual pesquisa vem elucidar e somar a uma nova perspectiva para prevenção à PE. Sugerem-se novos estudos de ensaios clínicos contemplando o duplo cego com um domínio mais direcionado à avaliação placentária.

Houve uma redução de 15,1 vezes no risco absoluto de PE com a utilização da metformina na população estudada, com a necessidade de tratar sete gestantes para evitar um desfecho.

\section{Notas}

Este estudo é parte da tese de doutorado em Saúde e Meio Ambiente de um dos autores (IBN) intitulada "Uso da metformina para prevenção dos desfechos obstétricos desfavoráveis em gestantes obesas", Universidade da Região de Joinville, 2018.

\section{Apoio financeiro}

Este estudo não recebeu apoio financeiro de fontes externas.

\section{Declaração de conflito de interesses}

Os autores declaram não haver conflitos de interesses relevantes ao conteúdo deste estudo.

\section{Contribuições dos autores}

Todos os autores fizeram contribuições substanciais para concepção, ou delineamento, ou aquisição, ou análise ou interpretação de dados; e redação do trabalho ou revisão crítica; e aprovação final da versão para publicação.

\section{Disponibilidade dos dados e responsabilidade pelos resultados}

Todos os autores declaram ter tido total acesso aos dados obtidos e assumem completa responsabilidade pela integridade destes resultados.

\section{Referências}

1. Costa AAR, Ribas MSSS, Amorim MMR SL. Mortalidade materna na cidade do Recife. Rev Bras Ginecol Obs 2002;24(7):455-62. https://doi.org/10.1590/ S0100-72032002000700005.
2. Adamo KB, Ferraro ZM, Goldfield G, Keely E, Stacey D, Hadjiyannakis S, Jean-Philippe S, Walker M, Barrowman NJ. The Maternal Obesity Management (MOM) Trial Protocol: a lifestyle intervention during pregnancy to minimize downstream obesity. Contemp Clin Trials 2013; 35(1):87-96. https://doi. org/10.1016/j.cct.2013.02.010.

3. World Health Organization. WHO Recommendations for Prevention and Treatment of Pre-Eclampsia and Eclampsia [monograph on the Internet]. WHO: Geneva, Switzerland; 2011. [cited 2020 Mar 20]. Available from: https://www.ncbi.nlm.nih.gov/books/NBK140555/.

4. Samiento-Piña M, Reyna-Villasmil E, Mejia-Montilla J, Santos-Bolivar J, Torres-Cepeda D, Reyna-Villasmil N. Valor predictivo de la proteinuria en 24 horas en la resultante neonatal de las preeclámpsicas. Clin Invest Ginecol Obstet 2017:44(3):119-124. https://doi. org/10.1016/j.gine.2015.10.006.

5. Nucci LB, Duncan BB, Mengue SS, Branchtein L, Shimidt MI FET. Assessment of weight gain during pregnancy in general prenatal care services in Brazil Avaliação de ganho ponderal intra-gestacional em serviços de assistência pré-natal no Brasil. Methods. Cad. Saúde Pública 2001:17(6):1367-74. https://doi. org/10.1590/S0102-311X2001000600007.

6. Stulbach TE, Benício MHD, Andreazza R, Kono S. Determinantes do ganho ponderal excessivo durante a gestação em serviço público de pré-natal de baixo risco. Rev Bras Epidemiol 2007;10:99-108. https://doi.org/10.1590/S1415-790X2007000100011.

7. Barton JR Sibai BM. Prediction and prevention of recurrent preeclampsia. Obstet Gynecol. Obs Gynecol 2008; 112:259-72. https://doi.org/10.1097/ AOG.0b013e $3181801 \mathrm{~d} 56$.

8. Bianco-Miotto T, Blundell C, Buckberry S, Chamley L, Chong S, Cottrell E, Dawson P. Hanna C, Holland O, Lewis RM, Moritz K, Myatt L, Perkins AV, Powell T, Saffery R, Sferruzzi-Perri A, Sibley C, Simmons D, O'Tierney-Ginn PF. IFPA meeting 2015 workshop report I: placental mitochondrial function, transport systems and epigenetics. Placenta 2016;48:3-6. https://doi.org/10.1016/j.placenta.2015.11.014.

9. Romero R, Erez O, Huttemann M, Panaitescu B, Conde-agudelo A, Pacora, P, Yoon BH, Grossman LI. Metformin, the aspirin of the 21st century: its role in gestational diabetes, prevention of preeclampsia and cancer, and the promotion of longevity. Am J Obs Gynecol 2017; 217(3):282-302. https://doi. org/10.1016/j.ajog.2017.06.003.

10. Dempsey JC, Williams M A., Leisenring WM, Shy K, Luthy DA. Maternal birth weight in relation to plasma lipid concentrations in early pregnancy. Am J Obstet Gynecol 2004; 190:1359-68. https://doi. org/10.1016/j.ajog.2003.10.710.

11. Silva JC, Amaral AR, Ferreira B da S, Petry Silva MR, Krelling PC. Obesidade durante a gravidez: resultados adversos da gestação e do parto. Rev. Bras. Ginecol. Obstet 2014; 36(11):509-13. https://doi. org/10.1590/S0100-720320140005024. 
12. Syngelaki A, Nicolaides KH, Balani J, Hyer S, Akolekar R, Kotecha R, Pastides A, Shehata H. Metformin versus Placebo in Obese Pregnant Women without Diabetes Mellitus. N Engl J Med 2016; 374(1):434-43. https://doi.org/10.1056/NEJMoa1509819.

13. Sibai BM. Hypertensive disorders of pregnancy: the United States perspective. Curr Opin Obstet Gynecol 2008; 20(2):102-06. https://doi.org/10.1097/ GCO.0b013e3282f73380.

14. Askie LM, Duley L, Henderson-Smart DJ, Stewart LA, PARIS Collaborative Group. Antiplatelet agents for prevention of pre-eclampsia: a meta-analysis of individual patient data. Lancet 2007: 369(9575):179198. https://doi.org/10.1016/S0140-6736(07)60712-0.

15. Rowan JA, Hague WM, Gao W, Battin MR, Moore MP. Metformin versus insulin for the treatment of gestational diabetes. N Engl J Med 2008; 358(19):2003-15. https://doi.org/10.1056/NEJMoa0707193.

16. Schulz KF, Altman DG, Moher D; CONSORT Group. CONSORT 2010 statement: updated guidelines for reporting parallel group randomised trials. BMJ 2010;340:c332. https://doi.org/10.1136/bmi.c332.

17. BRASIL. Ministério da Saúde. Secretaria de Atenção à Saúde. Departamento de Ações Programáticas Estratégicas. Gestação de alto risco: manual técnico [Imonograph on the Internet]. 5. ed. Brasilia: Editora do Ministério da Saúde; 2012a. [cited 2020 Mar 2]. Available from: http://bvsms.saude.gov.br/bvs/publicacoes/manual_tecnico_gestacao_alto_risco.pdf

18. Chiswick C, Reynolds RM, Denison F, Drake AJ, Forbes S, Newby DE, Walker BR, Quenby S, Wray S, Weeks A, Lashen H, Rodriguez A, Murray G, Whyte S Norman JE. Effect of metformin on maternal and fetal outcomes in obese pregnant women (EMPOWaR): a randomised, double-blind, placebo-controlled trial. Lancet Diabetes Endocrinol 2015:3(10):778-86. https://doi.org/10.1016/S2213-8587(15)00219-3.

19. Battaglia FC, Lubchenco LO. A practical classification of newborn infants by weight and gestational age. J Pediatr 1967:71(2):159-63. https://doi. org/10.1016/So022-3476(67)80066-0.

20. Fletcher RH, Fletcher SW, Fletcher GS. Epidemiologia clínica: elementos essenciais. 5.ed. Porto Alegre: ArtMed; 2014

21. Salihu HM, Lynch O, Alio AP, Kornosky JL, Clayton HB, Mbah AK. Extreme obesity and risk of placental abruption. Hum Reprod 2009; 24(2):438-44. https:// doi.org/10.1093/humrep/den421.

22. Chang JJ, Muglia LJ, Macones GA. Association of early-onset preeclampsia. In first pregnancy with normotensive second pregnancy outcomes: a population based study BJOG 2010;117(8):946-53. https:// doi.org/10.1111/j.1471-0528.2010.02594.X.
23. Reis ZSN, Lage EM, Teixeira PG, Porto LB, Guedes LR, Oliveira ECL, Cabral ACV. Pré-eclâmpsia precoce e tardia: uma classificação mais adequada para o prognóstico materno e perinatal? Rev Bras Ginecol e Obs 2010;32(12):584-90. https://doi.org/10.1590/ S0100-72032010001200004.

24. Zhao PL, Sheng XY, Zhou S, Yang T, Ma LY, Zhou Y Cui YM. Metformin versus insulin for gestational diabetes mellitus: a meta-analysis. Br J Clin Pharmacol 2015; 80(5):1224-34. https://doi.org/10.1111/bcp.12672.

25. Fujita D, Tanabe A, Sekijima T, Soen H, Narahara K, Yamashita Y, Soen H, Narahara K, Yamashita Y, Terai Y, Kamegai H, Ohmichi M. Role of extracellular signal-regulated kinase and AKT cascades in regulating hypoxia-induced angiogenic factors produced by a trophoblast-derived cell line. J Endocrinol. 2010; 206:131-40. https://doi.org/10.1677/JOE-10-0027.

26. Couto AA. Farmacologia Cardiovascular Aplicada à Clínica. ed. Revinterza edição, Rio de Janeiro; 1998. https://wwww.bibliomed.com.br/book/showchptrs. $\mathrm{cfm}$ ?bookid $=40 \&$ bookcatid $=3$

27. Bouchoucha M, Uzzan B, Cohen, R. Metformin and digestive disorders. Diabetes \& Metabolism. 2011;37(2):9096. https://doi.org/10.1016/j.diabet.2010.11.002.

28. Roberge S, Nicolaides K, Demers S, Hyett j, Chaillet N, Bujold E. The role of aspirin dose on the prevention of preeclampsia and fetal growth restriction: systematic review and meta-analysis. Am J Obstet Gynecol. 2017; 216(2):110-20. https://doi. org/10.1016/j.ajog.2016.09.076.

29. Norgard B, Puhó E, Czeizel AE, Skriver MV, Sorensen HT. Aspirin use during early pregnancy an the risk of congenital abnormalities: a population-based case-control study. Am J Obstet Gynecol. 2005;192:9223. https://doi.org/10.1016/j.ajog.2004.10.5.98.

30. Coomarasamy A, Honest H, Papaidannou S, Gee $\mathrm{H}$, Khan KS. Aspirin for prevention of preeclampsia in women with historical risk factors: a systematic review. Obstet Gynecol. 2003; 101:1319-32. https:// doi.org/10.1097/00006250-200306000-00030.

31. Briggs GG, Freeman RK, Yaffe SJ. (editors.). Drugs in pregnancy and lactation. 6th ed. Philadelphia: Lippincot Williams \& Wilkins; 2002.

32. Macedo PHG. Estudo morfológico de fetos e placentas de ratas sob a ação do etanol e/ou ácido acetilsalicilico [tese]. São Paulo: Universidade Federal de São Paulo, Escola Paulista de Medicina; 1998.

33. Duley L, Henderson-Smart DJ, Meher S, King JF. Antiplatelet agents for preventing pre-eclampsia and its complications. Cochrane Database Syst Rev [Internet]. 2007:[cited 2020 Mar 02];18(2). CD004659. Available from: https://doi.org/10.1002/14651858. CD004659.pub2. 
34. Rolnik DL, Wright D, Poon LCY, Syngelaki A, O'Gorman N, de Paco Matallana C. ASPRE trial: performance of screening for preterm pre-eclampsia. Ultrasound Obstet Gynecol. 2017:50(4):492-95. https://doi.org/10.1002/uog.18816.

35. Marchetti P, Del Guerra S, Marselli L, Lupi R, Masini M. Pollera M, Boggi U, Vistoli F, Del Prado FM. Pancreatic islets from type 2 diabetic patients have functional defects and increased apoptosis that are ameliorated by metformin. J Clin Endocrinol Metab.2004 89(11):5535-41. https://doi.org/10.1210/jc.2004-0150.

\section{Endereço para correspondência}

Iramar Baptistella do Nascimento

Universidade do Estado de Santa Catarina

Rua Luiz Fernando Hastreiter, 180, Predio 1, Laboratório de Ergonomia, sala 26

Centenário, 89.283-081

São Bento do Sul, SC, Brasil 